\title{
Quantization of gauge-invariant theories through the Dirac-bracket formalism
}

\author{
M. E. V. Costa and H. O. Girotti \\ Instituto de Física, Universidade Federal do Rio Grande do Sul, 90000 Porto Alegre, RS, Brasil
}

(Received 10 December 1980)

\begin{abstract}
A physical system possessing first-class constraints is quantized by using the Dirac-bracket formalism. The main features of this quantization procedure are analyzed. The model enables us to present a situation where gaugedependent Dirac brackets give origin to gauge-independent equations of motion.
\end{abstract}

As is known, gauge theories fall into the class of constrained systems..$^{1-3}$ The proposal of Dirac ${ }^{1}$ for quantizing such systems consists of imposing the first-class constraints as restrictions on the Hilbert space of state vectors while the quantummechanical commutators are directly read off from the corresponding Poisson brackets.

On the other hand, it has been recognized that the first-class constraints together with the subsidiary (gauge) conditions form a set of secondclass constraints. ${ }^{3}$ An alternative scheme for quantizing a gauge theory emerges from this observation. In fact, one can quantize the theory by abstracting the quantum-mechanical commutators from the corresponding Dirac brackets while constraints and subsidiary conditions translate into strong operator identities. We shall refer to this quantization procedure as to the Dirac-bracket quantization procedure (DBQP).

Since the DBQP is not a widespread technique among physicists we attempt to illustrate in this paper its use and consequences. ${ }^{4}$ This investigation will be carried out at the level of the operator approach and in connection with a simple, but sufficiently illuminating, nonrelativistic gauge-invariant model put forward by Christ and Lee for another purpose. ${ }^{5}$

Through this simple model we shall also learn about an interesting, and we believe new, situation which can arise when a gauge-invariant theory is quantized by using the DBQP. Indeed, what one expects from general considerations is a set of equations of motion and of Dirac brackets depending on the choice of gauge. ${ }^{3}$ However, in the present case only the Dirac brackets turn out to be gauge-dependent entities.

The classical dynamics of the model is described by the Lagrangian

$$
\begin{aligned}
L= & \frac{1}{2}\left(\dot{x}_{1}{ }^{2}+\dot{x}_{2}{ }^{2}\right)-\left(x_{1} \dot{x}_{2}-x_{2} \dot{x}_{1}\right) x_{3} \\
& +\frac{1}{2} x_{3}{ }^{2}\left(x_{1}{ }^{2}+x_{2}{ }^{2}\right)-V\left(x_{1}{ }^{2}+x_{2}{ }^{2}\right),
\end{aligned}
$$

where $x_{1}, x_{2}$, and $x_{3}$ are Cartesian coordinates and the overdot indicates differentiation with respect to time.
From the analysis of the matrix $\left\|\partial^{2} L / \partial \dot{x}_{k} \partial \dot{x}_{j}\right\|$, $k, j=1,2,3$, one concludes that (1) is a singular system. ${ }^{1,6}$ It possesses a primary constraint

$$
\psi^{1}(x, p) \equiv p_{3} \approx 0,
$$

and a secondary constraint

$$
\psi^{2}(x, p) \equiv x_{1} p_{2}-x_{2} p_{1} \approx 0 .
$$

Here $p_{k} \equiv \partial L / \partial \dot{x}_{k}$ denotes the momentum canonically conjugate to $x_{k}$. The sign of weak equality is being used in the sense of Dirac. ${ }^{1}$ One can convince oneself that there are no further constraints. This is essentially due to the particular functional dependence assumed for the potential $V=V\left(x_{1}{ }^{2}+x_{2}{ }^{2}\right)$. Since the Poisson brackets

$$
\left[\psi^{1}, \psi^{2}\right]=0,
$$

$\psi^{1}$ and $\psi^{2}$ are first-class constraints. Furthermore, the total Hamiltonian of the system is found to read

$$
H_{T}=H_{M}+\xi_{1} \psi^{1}+\xi_{2} \psi^{2},
$$

where

$$
H_{M}=\frac{1}{2} p_{1}{ }^{2}+\frac{1}{2}{p_{2}}^{2}+V\left(x_{1}{ }^{2}+x_{2}{ }^{2}\right),
$$

and $M$ is a subspace of phase space in which all constraints hold..$^{6,7}$ The $\xi$ 's are the so-far-undetermined Lagrange multipliers.

To solve for the $\xi$ 's, and therefore for the classical dynamics, one must specify a number of subsidiary (gauge) conditions equal to the number of first-class constraints. ${ }^{2,3,7}$ We choose

$$
\begin{aligned}
& \psi^{3}(x, p) \equiv b-c \arctan \frac{x_{2}}{x_{1}} \approx 0, \\
& \psi^{4}(x, p) \equiv x_{3} \approx 0,
\end{aligned}
$$

where $b$ and $c$ are nonzero constants. As required $\mathrm{d}^{2,3,7}$

$$
\operatorname{det}\|Q\|=c^{2}>0,
$$

where $\|Q\|$ is a matrix whose elements are given by

$$
Q^{a b}=\left[\psi^{a}, \psi^{b}\right], \quad a, b=1,2,3,4 .
$$


Clearly, det $\|Q\|$ is the square of the Faddeev-Popov determinant. ${ }^{2}$ One should notice that Eq. (6a) defines different gauges for different values of the constants $b$ and $c$. Indeed, Eqs. (6) define a set of gauges.

To complete the classical description of the system one must find the equations governing the time evolution of $\xi, x$, and $p$. By demanding persistence in time of constraints and gauge conditions one obtains

$$
\xi_{a}=-\left.\sum_{b=1}^{4} R_{a b}\left[\psi^{b}, H_{M}\right]\right|_{\Gamma} *=0
$$

where $\|R\|$ is the inverse of $\|Q\|$ and $\Gamma^{*}$ is the submanifold of $M\left(\Gamma^{*} \subset M\right)$ in which all constraints and subsidiary conditions hold.

The result (9) is, as we show next, at the root of the above-mentioned peculiar situation.

At the classical level Eq. (9) tell us that the Dirac brackets $\mathrm{s}^{1-3,7}$ of any function $f$ of $x$ and $p$ with $H_{M}$ reduces in $\Gamma^{*}$ to its corresponding Poisson brackets, i.e.,

$$
\begin{aligned}
{\left.\left[f, H_{M}\right]_{\mathrm{DB}}\right|_{\Gamma} * } & =\left.\left\{\left[f, H_{M}\right]-\sum_{a, b=1}^{4}\left[f, \psi^{a}\right] R_{a b}\left[\psi^{b}, H_{M}\right]\right\}\right|_{\Gamma^{*}} \\
& =\left.\left[f, H_{M}\right]\right|_{\Gamma^{*}}
\end{aligned}
$$

Then, within the set of gauges defined by Eqs. (6), the equations of motion

$$
\begin{aligned}
& \dot{x}_{r}=\left.\left[x_{r}, H_{M}\right]_{\mathrm{DB}}\right|_{\Gamma *}=p_{r}, \\
& \dot{p}_{r}=\left.\left[p_{r}, H_{M}\right]_{\mathrm{DB}}\right|_{\Gamma} *=-\frac{\partial V}{\partial x_{r}},
\end{aligned}
$$

$r=1,2$, turn out to be gauge independent. On the other hand, the Dirac brackets

$$
\begin{aligned}
& {\left.\left[x_{1}, p_{1}\right]_{\mathrm{DB}}\right|_{\Gamma} *=\frac{1}{1+e^{2}},\left.\left[x_{1}, p_{2}\right]_{\mathrm{DB}}\right|_{\Gamma} *=\frac{e}{1+e^{2}},} \\
& {\left.\left[x_{2}, p_{1}\right]_{\mathrm{DB}}\right|_{\Gamma} *=\frac{e}{1+e^{2}},\left.\left[x_{2}, p_{2}\right]_{\mathrm{DB}}\right|_{\Gamma} *=\frac{e^{2}}{1+e^{2}},}
\end{aligned}
$$

where

$$
e=\tan \frac{b}{c}
$$

do depend upon the gauge. One also verifies that

$$
\left.\left[x_{r}, x_{s}\right]_{\mathrm{DB}}\right|_{\Gamma^{*}}=\left.\left[p_{r}, p_{s}\right]_{\mathrm{DB}}\right|_{\Gamma^{*}}=0, r, s=1,2 \text {. }
$$

From a physical point of view these results can be easily interpreted. All one is doing is describing the two-dimensional motion $\left(x_{3}=0\right)$ of a unit mass particle which is constrained to move with zero angular momentum. Therefore, the particle trajectory must be a straight line passing through the origin of coordinates. The gauge condition (6a) just specifies the slope of the trajectory. Since the potential $V$ only depends upon the absolute distance $\left(x_{1}{ }^{2}+x_{2}{ }^{2}\right)$ one cannot detect any change in the slope of the trajectory by looking at the structure of the equations of motion.

We shall turn next to the problem of quantizing the system. According to the DBQP one is to abstract the quantum-mechanical commutators from the corresponding Dirac brackets, with the constraints and subsidiary conditions being understood as strong equations. Then, in view of Eqs. (2), (5), (6), (12), (13), and (14), we take $(\hbar=1)$

$$
\hat{H}=\frac{1}{2}{\hat{p}_{1}}^{2}+\frac{1}{2} \hat{p}_{2}{ }^{2}+\hat{V}\left(\hat{x}_{1}{ }^{2}+\hat{x}_{2}{ }^{2}\right)
$$

as the Hamiltonian operator, and

$$
\begin{aligned}
& \hat{\phi} \equiv \hat{x}_{1} \hat{p}_{2}-\hat{x}_{2} \hat{p}_{1}=0, \\
& \hat{\eta} \equiv \hat{x}_{2}-e \hat{x}_{1}=0
\end{aligned}
$$

as strong operator equations. Furthermore,

$$
\begin{aligned}
& {\left[\hat{x}_{r}, \hat{x}_{s}\right]=\left[\hat{p}_{r}, \hat{p}_{s}\right]=0, \quad r, s=1,2,} \\
& {\left[\hat{x}_{1}, \hat{p}_{1}\right]=i \frac{1}{1+e^{2}}, \quad\left[\hat{x}_{1}, \hat{p}_{2}\right]=i \frac{e}{1+e^{2}},} \\
& {\left[\hat{x}_{2}, \hat{p}_{1}\right]=i \frac{e}{1+e^{2}}, \quad\left[\hat{x}_{2}, \hat{p}_{2}\right]=i \frac{e^{2}}{1+e^{2}} .}
\end{aligned}
$$

From (16) and (18) it follows that $\hat{\phi}^{\dagger}=\hat{\phi}$. Obviously, $\hat{\eta}^{\dagger}=\hat{\eta}$.

It is now a simple exercise to verify that the Heisenberg equations of motion

$$
\begin{aligned}
& \dot{\hat{x}}_{r}=i\left[\hat{H}, \hat{x}_{r}\right], \quad r=1,2, \\
& \dot{\hat{p}}_{r}=i\left[\hat{H}, \hat{p}_{r}\right],
\end{aligned}
$$

together with Eqs. (16)-(18), lead one to

$$
\begin{aligned}
& \dot{\hat{x}}_{r}=\hat{p}_{r}, \quad r=1,2, \\
& \dot{\hat{p}}_{r}=-\frac{\partial \hat{V}}{\partial \hat{x}_{r}}, \quad r=1,2 .
\end{aligned}
$$

This last set of equations is formally identical to (11).

The consistency of the DBQP can be easily checked by recalling that if one succeeds in finding a formulation of the dynamics in terms of independent (unconstrained) variables, such variables must obey the standard canonical commutation relation. ${ }^{3}$ In the present case it is very easy to formulate the dynamics in terms of independent variables because as a result of the constraints and gauge conditions one is left with a one-dimensional system. After defining the Cartesian coordinate $^{8}$

$$
x^{*} \equiv\left[+\left(1+e^{2}\right)^{1 / 2}\right] x_{1},
$$


and its corresponding momentum

$$
p^{*} \equiv\left[+\left(1+e^{2}\right)^{1 / 2}\right] p_{1},
$$

it follows from (18) that, as required,

$$
\left[\hat{x}^{*}, \hat{p}^{*}\right]=i \text {. }
$$

Furthermore, in terms of $\hat{x}^{*}$ and $\hat{p}^{*}$ the Hamiltonian operator reduces to

$$
\hat{H}^{*}=\frac{1}{2} \hat{p}^{* 2}+\hat{V}\left(\hat{x}^{* 2}\right),
$$

as is also required..$^{*}$ and $p^{*}$ are phase-space coordinates spanning $\Gamma^{*}$.

We believe that our purpose of carrying out a clear and complete application of the DBQP has been achieved. As a by-product of our work we have found that there exists a situation, which was not previously contemplated in the literature, ${ }^{1-3,7}$ in which a continuous gauge symmetry gives origin to a continuous infinite set of commutation rules but produces no effect whatsoever on the equations of motion. This effect is not to be confused with the real ambiguities arising in the canonical Hamiltonian quantization procedure..$^{9}$

The work of H.O.G. was supported in part by Conselho Nacional de Desenvolvimento Científico e Tecnológico (CNPq) and Financiadora de Estudos e Projetos (FINEP), Brasil.
${ }^{1}$ P. A. M. Dirac, Lectures on Quantum Mechanics, Yeshiva University-Belfer Graduate School of Science (Academic, New York, 1964).

${ }^{2}$ L. D. Faddeev, Teor. Mat. Fiz. 1, 3 (1969) [Theor. Math. Phys. (USSR) 1,1 (1970)].

${ }^{3}$ E. S. Fradkin and G. A. Vilkovisky, Report No. CERN TH. 2332, 1977 (unpublished).

${ }^{4}$ The quantization of electrodynamics by using the DBQP can be found in H. O. Girotti and K. D. Rothe, Lett. Nuovo Cimento 31, 545 (1981).

${ }^{5}$ N. H. Christ and T. D. Lee, Phys. Rev. D $\underline{22}, 939$ (1980).
${ }^{6}$ E. C. G. Sudarshan and N. Mukunda, Classical Dynamics: A Modern Perspective (Wiley, New York, 1974). ${ }^{7}$ P. Senjanovic, Ann. Phys. (N.Y.) 100, 227 (1976).

${ }^{8}$ One is not to confuse $x^{*}$ with the plane polar coordinate

$$
\begin{aligned}
r & =+\left(x_{1}{ }^{2}+x_{2}{ }^{2}\right)^{1 / 2} \\
& =\left[+\left(1+e^{2}\right)^{1 / 2}\right]\left[+\left(x_{1}{ }^{2}\right)^{1 / 2}\right] .
\end{aligned}
$$

${ }^{9}$ A. P. Balachandran, T. R. Govindarajan, and B. Vijayalakshmi, Phys. Rev. D 18, 1950 (1978) and references therein. 